\title{
Solução da Equação Transiente de Transporte em Domínio Semi-infinito pela Combinação dos Métodos Espectral e $\operatorname{LTS}_{\mathrm{N}}$
}

J.V.P. de OLIVEIRA ${ }^{1}$, Departamento de Matemática, UFSM, Av. Roraima, S/N, CCNE, 97105-900 Santa Maria, RS, Brasil

A.V. CARDONA2, FAMAT, PUCRS, Prédio 15, Av. Ipiranga, 6681, 90619900 Porto Alegre, RS, Brasil.

\begin{abstract}
Resumo. Neste trabalho, apresentamos uma nova abordagem para solução da equação de transporte linear dependente do tempo em domínios unidimensionais ilimitados, combinando o método espectral e o método LTS $_{N}$. Para tanto, usando a idéia do método espectral, expandimos o fluxo angular numa série truncada de polinômios de Laguerre na variável temporal, tomamos momentos, obtendo, assim, um conjunto de problemas estacionários de transporte, que são resolvidos recurssivamente pelo método $\mathrm{LTS}_{\mathrm{N}}$. Resultados numéricos são apresentados.
\end{abstract}

\section{Introdução}

Recentemente, Oliveira [1] propôs uma nova abordagem para resolver problemas unidimencionais de transporte com dependência temporal, combinando os métodos espectral [2] e $\operatorname{LTS}_{\mathrm{N}}$ [3]. Essa formulação tem sido aplicada com sucesso na solução de problemas de transporte dependentes do tempo em domínios limitados, tanto lineares $[4,5]$, quanto não lineares $[6,7]$. Neste trabalho, propomos estender essa formulação à solução de problemas transientes de transporte em domínios unidimencionais ilimitados.

Com o objetivo de apresentar as idéias propostas, consideremos o problema transiente monoenergético de ordenadas discretas em uma dimensão espacial, com espalhamento isotrópico, numa placa, descrito pelas equações $S_{N}$

$$
\begin{gathered}
\frac{\partial}{\partial t} \Psi_{n}(x, t)+\mu_{n} \frac{\partial}{\partial x} \Psi_{n}(x, t)+\Psi_{n}(x, t)=\frac{c}{2} \sum_{k=1}^{N} \omega_{k} \Psi_{k}(x, t), \\
\Psi_{n}(0, t)=1, \text { se } n=1, \ldots, \frac{N}{2},
\end{gathered}
$$

\footnotetext{
${ }^{1}$ pardal@ccne.ufsm.br

2 acardona@mat.pucrs.br
} 


$$
\lim _{x \rightarrow \infty} \Psi_{n}(x, t)=0
$$

$$
\Psi_{n}(x, t)=0, \text { para } t<0,
$$

onde $\Psi_{n}(x, t)$ denota o fluxo de nêutrons na posição $x$, na direção discreta $\mu_{n}$, no instante $t$. Aqui, as direções discretas $\mu_{n}$, ordenadas de forma decrescente e os coeficientes $\omega_{n}$, com $n=1,2, \ldots, N$, são, respectivamente, as raízes e os pesos da quadratura de Gauss-Legendre.

\section{O Método Espectral}

Para resolver o problema descrito pelas equações $(1.1-1.3)$, inicialmente fazemos uso do método espectral. Para isso, expandimos o fluxo angular discreto $\Psi_{n}(x, t)$ em uma série truncada de polinômios de Laguerre [8] na variável $t$, que são ortonormais em relação à função peso $e^{-t}$ sobre o intervalo $[0,+\infty)$, isto é,

$$
\Psi_{n}(x, t)=\sum_{k=0}^{M} \Psi_{n}^{k}(x) L_{k}(t)
$$

tal que

$$
\int_{0}^{+\infty} e^{-t} L_{k}(t) L_{m}(t) d t=\left\{\begin{array}{l}
1, \text { se } k=m \\
0, \text { se } k \neq m
\end{array},\right.
$$

onde $L_{k}(z)$ denota o polinômio de Laguerre de grau $k, k=0,1, \ldots, M$.

Substituindo a aproximação dada pela equação $(2.1)$ nas equações $(1.1-1.3)$, multiplicando as equações resultantes por $e^{-t} L_{m}(t)$ e, em seguida, integrando o resultado em $0<t<+\infty$, fazendo uso das propriedades de ortogonalidade dos polinômios de Laguerre, obtemos:

$$
\begin{gathered}
\mu_{n} \frac{d}{d x} \Psi_{n}^{m}(x)+2 \Psi_{n}^{m}(x)=\frac{c}{2} \sum_{k=1}^{N} \omega_{k} \Psi_{n}^{m}(x)-\sum_{i=0}^{m-1} \Psi_{n}^{i}(x), \\
\Psi_{n}^{m}(0)=\delta_{m, 0}, \text { para } n=1, \ldots, \frac{N}{2},
\end{gathered}
$$

$$
\lim _{x \rightarrow \infty} \Psi_{n}^{m}(x)=0
$$

onde $\delta_{m, 0}$ denota o delta de Kronecker. Cabe enfatizar que, na obtenção da equação (2.3), fizemos uso da expressão resultante da substituição da expansão (2.1) na equação (1.4), utilizando procedimento análogo ao usado em $[1,4,5]$.

Portanto, uma vez determinada cada componente $\Psi_{n}^{m}(x)$, para $m=0,1, \ldots, M$, no problema diferencial $(2.3-2.5)$, o fluxo angular discreto fica completamente estabelecido pela equação (2.1). 


\section{O Método $\operatorname{LTS}_{\mathrm{N}}$}

Basicamente, esse método consiste em aplicar a transformada de Laplace nas equações $S_{N}$, resolver o sistema algébrico resultante da aplicação da transformada de Laplace e inverter analiticamente o fluxo angular transformado. Maiores detalhes sobre o método LTS $_{\mathrm{N}}$, podem ser obtidos no artigo de Segatto e Vilhena [3].

Assim, aplicando a transformada de Laplace na equação (2.3) com relação a variável espacial $x$, obtemos, para cada $m=0,1, \ldots, M$, a equação matricial

$$
(s I-A) \bar{\Psi}_{m}(s)=\Psi_{m}(0)-U^{-1} \sum_{i=0}^{m-1} \bar{\Psi}_{i}(s)
$$

onde $I$ representa a matriz identidade de ordem $N$ e $U$ denota a matriz diagonal tendo como elementos as raízes $\mu_{n}$. Os elementos da matriz $A$ são

$$
a_{i, j}=\left\{\begin{array}{l}
\frac{\omega_{j}}{2 \mu_{i}} c-\frac{2}{\mu_{i}}, \text { se } i=j \\
\frac{\omega_{j}}{2 \mu_{i}} c, \text { se } i \neq j
\end{array} .\right.
$$

Enquanto que, $\bar{\Psi}_{m}(s)$ e $\Psi_{m}(0)$ denotam, respectivamente, os vetores

$$
\bar{\Psi}_{m}(s)=\left[\begin{array}{llll}
\bar{\Psi}_{1}^{m}(s) & \bar{\Psi}_{2}^{m}(s) & \ldots & \bar{\Psi}_{N}^{m}(s)
\end{array}\right]^{T}
$$

$$
\Psi_{m}(0)=\left[\begin{array}{lllll}
\Psi_{1}^{m}(0) & \Psi_{2}^{m}(0) & \ldots & \Psi_{N}^{m}(0)
\end{array}\right]^{T}
$$

Na resolução da equação matricial (3.1), utilizamos o processo de diagonalização da matriz $A$ apresentado em [3],

$$
A=V D V^{-1}
$$

onde $V$ e $D$ são, respectivamente, as matrizes dos autovetores e autovalores de $A$. E, em seguida, aplicamos a transformada inversa de Laplace na equação resultante. Desta forma, utilizando a condição de contorno dada pela equação (2.4), obtemos a seguinte expressão recursiva para os momentos do fluxo angular discreto,

$$
\begin{aligned}
\Psi_{n}^{m}(x)= & \sum_{i=1}^{N} v(n, i) e^{d(i) x}\left\{\sum_{j=1}^{\frac{N}{2}}\left[v^{-1}(i, j) \delta_{m, 0}+v^{-1}\left(i, j+\frac{N}{2}\right) \Psi_{j+\frac{N}{2}}^{m}(0)\right]\right. \\
& \left.-\sum_{j=1}^{N} \frac{v^{-1}(i, j)}{\mu_{j}} \sum_{k=0}^{m-1} \int_{0}^{x} e^{-d(i) \tau} \Psi_{j}^{k}(\tau) d \tau\right\}
\end{aligned}
$$

sendo $d(i), v(i, j)$ e $v^{-1}(i, j)$, respectivamente, os elementos das matrizes $D, V$ e $V^{-1}$. Aqui os autovalores $d(i)$ estão ordenados de forma ascendente. 
Analisando a estrutura da equação (3.6) e considerando a equação (2.5), percebese que os coeficientes das funções $e^{d(i) x}$, para $i=\left(\frac{N}{2}+1\right), \ldots, N$, devem ser nulos. Então, concluimos que o fluxo angular discreto, $\Psi_{n}^{m}(x)$, tem por solução a seguinte forma fechada,

$$
\Psi_{n}^{m}(x)=\sum_{i=1}^{\frac{N}{2}}\left(\sum_{\gamma=0}^{m} \varphi_{n}^{m}(i, \gamma) \frac{x^{\gamma}}{\gamma !}\right) e^{d(i) x}
$$

onde os elementos $\varphi_{n}^{m}(i, \gamma)$ são construídos recursivamente, para os índices $m$, através da relação

$$
\varphi_{n}^{0}(i, 0)=v(n, i) \sum_{j=1}^{\frac{N}{2}}\left[v^{-1}(i, j)+v^{-1}\left(i, j+\frac{N}{2}\right) \Psi_{j+\frac{N}{2}}^{0}(0)\right]
$$

e

$$
\begin{aligned}
\varphi_{n}^{m}(i, 0)= & v(n, i) \sum_{j=1}^{\frac{N}{2}} v^{-1}\left(i, j+\frac{N}{2}\right) \Psi_{j+\frac{N}{2}}^{m}(0) \\
& +\sum_{j=1}^{N} \sum_{k=0}^{m-1} \sum_{\lambda=0}^{k} \frac{1}{\mu_{j}}\left[\sum_{\substack{\zeta=1 \\
\zeta \neq 1}}^{N} \frac{v(n, \zeta) v^{-1}(\zeta, j) \varphi_{j}^{k}(i, \lambda)}{(d(\zeta)-d(i))^{\gamma+1}}\right. \\
& -\sum_{\substack{\zeta=1 \\
\zeta \neq 1}}^{\frac{N}{2}} \frac{v(n, i) v^{-1}(i, j) \varphi_{j}^{k}(\zeta, \lambda)}{\left.(d(i)-d(\zeta))^{\gamma+1}\right]} \\
\varphi_{n}^{m}(i, \gamma)= & \sum_{j=1}^{N} \frac{1}{\mu_{j}}\left[\sum_{k=\gamma}^{m-1} \sum_{\substack{\zeta=1 \\
\zeta \neq 1}}^{\frac{N}{2}} \frac{v(n, \zeta) v^{-1}(\zeta, j)}{(d(\zeta)-d(i))^{\gamma+1} \sum_{\lambda=\gamma}^{k}} \frac{\varphi_{j}^{k}(i, \lambda)}{(d(\zeta)-d(i))^{-\lambda}}\right. \\
& \left.-\sum_{k=\gamma-1}^{m-1} v(n, i) v^{-1}(i, j) \varphi_{j}^{k}(i, \lambda-1)\right]
\end{aligned}
$$

para $\gamma=1,2, \ldots,(m-1)$

$$
\varphi_{n}^{m}(i, m)=-\sum_{j=1}^{N} \frac{v(n, i) v^{-1}(i, j) \varphi_{j}^{m-1}(i, m-1)}{\mu_{j}},
$$

para $m \neq 0$. As incógnitas $\Psi_{j+\frac{N}{2}}^{m}(0)$, para $j=1,2, \ldots, \frac{N}{2}$, são determinadas, para cada $m$, através da solução do seguinte conjunto de equações lineares 


$$
\sum_{j=1}^{\frac{N}{2}}\left[v^{-1}(i, j)+v^{-1}\left(i, j+\frac{N}{2}\right) \Psi_{j+\frac{N}{2}}^{0}(0)\right]=0
$$

$\operatorname{com} i=\left(\frac{N}{2}+1\right), \ldots, N$, para $m=0, \mathrm{e}$

$$
\sum_{j=1}^{\frac{N}{2}}\left[v^{-1}\left(i, j+\frac{N}{2}\right) \Psi_{j+\frac{N}{2}}^{m}(0)\right]=\sum_{\zeta=1}^{\frac{N}{2}} \sum_{j=1}^{N} \frac{v^{-1}(i, j)}{\mu_{j}} \sum_{k=0}^{m-1} \sum_{\gamma=0}^{k} \frac{\varphi_{j}^{k}(\zeta, \gamma)}{(d(i)-d(\zeta))^{\gamma+1}}
$$

$\operatorname{com} i=\left(\frac{N}{2}+1\right), \ldots, N$, para $m \neq 0$.

Finalmente, determinados os coeficientes $\Psi_{j+\frac{N}{2}}^{m}(0)$ no sistema de equações $(3.12-$ $3.13)$ e, então, os coeficientes $\varphi_{n}^{m}(i, \gamma)$ nas equações $(3.8-3.11)$, o fluxo angular discreto fica completamente estabelecido pelas equações (2.1) e (3.7).

\section{Resultados Numéricos e Conclusões}

Com o objetivo de testar o método proposto, sob o ponto de vista da modelagem computacional, consideramos o problema descrito pelas equações $(1.1-1.4)$. $\mathrm{Na}$ Tabela 1 apresentamos os resultados das simulações numéricas para o fluxo escalar descrito por

$$
\Phi(x, t)=\sum_{n=1}^{N} \omega_{n} \Psi_{n}(x, t)
$$

em $x=0, t=1.0, c=0.1, N=2,4,6$ e 8, e $M$ variando de 0 a 18 . Enquanto que, na Tabela 2 , apresentamos os valores para o fluxo escalar em $t=8.0$, considerando $c=0.1, N=8, x=5,6,7$ e 8 , e $M$ variando de 0 a 18 .

Na Tabela 1 pode-se observar a convergência numérica do método, tanto com a variação de $M$ (ordem da aproximação em polinômios de Laguerre) quanto com a variação de $N$ (ordem da quadratura de Gauss-Legendre). 


\begin{tabular}{|c|c|c|c|c|}
\hline$M$ & $N=2$ & $N=4$ & $N=6$ & $N=8$ \\
\hline 0 & 1.012822 & 1.012822 & 1.012822 & 1.012822 \\
1 & 1.012822 & 1.012822 & 1.012822 & 1.012822 \\
2 & 1.014424 & 1.014424 & 1.014424 & 1.014424 \\
3 & 1.015464 & 1.015464 & 1.015464 & 1.015464 \\
4 & 1.015938 & 1.015938 & 1.015938 & 1.015938 \\
5 & 1.016111 & 1.016111 & 1.016111 & 1.016111 \\
6 & 1.016157 & 1.016157 & 1.016157 & 1.016157 \\
7 & 1.016161 & 1.016161 & 1.016161 & 1.016161 \\
8 & 1.016154 & 1.016154 & 1.016154 & 1.016154 \\
9 & 1.016148 & 1.016148 & 1.016148 & 1.016148 \\
10 & 1.016144 & 1.016144 & 1.016144 & 1.016144 \\
11 & 1.016141 & 1.016141 & 1.016141 & 1.016141 \\
12 & 1.016140 & 1.016140 & 1.016140 & 1.016140 \\
13 & 1.016140 & 1.016140 & 1.016140 & 1.016140 \\
14 & 1.016139 & 1.016139 & 1.016139 & 1.016139 \\
15 & 1.016139 & 1.016139 & 1.016139 & 1.016139 \\
16 & 1.016139 & 1.016139 & 1.016139 & 1.016139 \\
17 & 1.016139 & 1.016139 & 1.016139 & 1.016139 \\
18 & 1.016139 & 1.016139 & 1.016139 & 1.016139 \\
\hline
\end{tabular}

Tabela 1: Fluxo escalar em $x=0$, no instante $t=1.0, c=0.1$.

Convém salientar que nenhuma aproximação foi feita no processo de obtenção da solução, exceto pelo truncamento da expansão em polinômios de Laguerre na variável temporal. Com facilidade esta formulação pode ser adequada para resolver outros problemas, tais como: problemas anisotrópicos (pois esta metodologia não altera a estrutura do núcleo de espalhamento), problemas com outros tipos de condições de contorno e iniciais.

Ademais, o esforço computacional dispensado na obtenção dos resultados numéricos é ínfimo. Isso se deve, em parte, ao fato de termos calculado analiticamente a integral de convolução proveniente da aplicação da transformada de Laplace inversa na equação matricial (3.1). Para a ordem de quadratura $N=8$ e a aproximação em polinômios de Laguerre $M=18$, os valores numéricos foram gerados em $2.19 \mathrm{~s}$. As simulações numéricas foram realizadas em um computador Pentium III - $450 \mathrm{MHz}$, com $64 \mathrm{MB}$ de memória RAM.

\section{Agradecimentos}

Os autores agradecem a FAPERGS pelo suporte finaceiro parcial na elaboração deste trabalho. 


\begin{tabular}{|c|c|c|c|c|}
\hline$M$ & $x=5$ & $x=6$ & $x=7$ & $x=8$ \\
\hline 0 & $7.058294 \mathrm{E}-05$ & $1.369089 \mathrm{E}-05$ & $2.706326 \mathrm{E}-06$ & $5.430273 \mathrm{E}-07$ \\
1 & $1.756194 \mathrm{E}-03$ & $4.001721 \mathrm{E}-04$ & $9.077994 \mathrm{E}-05$ & $2.054662 \mathrm{E}-05$ \\
2 & $6.339704 \mathrm{E}-03$ & $1.741232 \mathrm{E}-03$ & $4.620548 \mathrm{E}-04$ & $1.197059 \mathrm{E}-04$ \\
3 & $7.195742 \mathrm{E}-03$ & $2.211224 \mathrm{E}-03$ & $6.507256 \mathrm{E}-04$ & $1.853569 \mathrm{E}-04$ \\
4 & $7.640165 \mathrm{E}-03$ & $2.291025 \mathrm{E}-03$ & $6.446690 \mathrm{E}-04$ & $1.726433 \mathrm{E}-04$ \\
5 & $7.651385 \mathrm{E}-03$ & $2.341734 \mathrm{E}-03$ & $6.688867 \mathrm{E}-04$ & $1.799629 \mathrm{E}-04$ \\
6 & $7.781496 \mathrm{E}-03$ & $2.388237 \mathrm{E}-03$ & $6.750379 \mathrm{E}-04$ & $1.776835 \mathrm{E}-04$ \\
7 & $7.791235 \mathrm{E}-03$ & $2.388543 \mathrm{E}-03$ & $6.740498 \mathrm{E}-04$ & $1.771983 \mathrm{E}-04$ \\
8 & $7.785852 \mathrm{E}-03$ & $2.396551 \mathrm{E}-03$ & $6.774742 \mathrm{E}-04$ & $1.776961 \mathrm{E}-04$ \\
9 & $7.796911 \mathrm{E}-03$ & $2.402772 \mathrm{E}-03$ & $6.781062 \mathrm{E}-04$ & $1.772208 \mathrm{E}-04$ \\
10 & $7.800231 \mathrm{E}-03$ & $2.403295 \mathrm{E}-03$ & $6.778662 \mathrm{E}-04$ & $1.770812 \mathrm{E}-04$ \\
11 & $7.797908 \mathrm{E}-03$ & $2.403622 \mathrm{E}-03$ & $6.781963 \mathrm{E}-04$ & $1.771419 \mathrm{E}-04$ \\
12 & $7.797155 \mathrm{E}-03$ & $2.404500 \mathrm{E}-03$ & $6.784306 \mathrm{E}-04$ & $1.771037 \mathrm{E}-04$ \\
13 & $7.797675 \mathrm{E}-03$ & $2.404915 \mathrm{E}-03$ & $6.784277 \mathrm{E}-04$ & $1.770563 \mathrm{E}-04$ \\
14 & $7.797807 \mathrm{E}-03$ & $2.404941 \mathrm{E}-03$ & $6.784158 \mathrm{E}-04$ & $1.770510 \mathrm{E}-04$ \\
15 & $7.797550 \mathrm{E}-03$ & $2.404953 \mathrm{E}-03$ & $6.784443 \mathrm{E}-04$ & $1.770545 \mathrm{E}-04$ \\
16 & $7.797363 \mathrm{E}-03$ & $2.405012 \mathrm{E}-03$ & $6.784661 \mathrm{E}-04$ & $1.770508 \mathrm{E}-04$ \\
17 & $7.797332 \mathrm{E}-03$ & $2.405054 \mathrm{E}-03$ & $6.784692 \mathrm{E}-04$ & $1.770464 \mathrm{E}-04$ \\
18 & $7.797345 \mathrm{E}-03$ & $2.405064 \mathrm{E}-03$ & $6.784675 \mathrm{E}-04$ & $1.770451 \mathrm{E}-04$ \\
\hline
\end{tabular}

Tabela 2: Fluxo escalar no instante $t=8.0, c=0.1$ e $N=8$.

\begin{abstract}
In this work we report a solution for the time-dependent transport equation in an unbounded onedimensional slab by th combined spectral and LTS $_{\mathrm{N}}$ methods. To this and, we replace the truncated series in Laguerre polynomial for the angular flux in the time-dependent transport equation, take moments and solve the resulting set of one-dimensional problems by the $\operatorname{LTS}_{N}$ method. We present numerical simulations.
\end{abstract}

\title{
Referências
}

[1] J.V.P. de Oliveira, "Solução da Equação de Transporte Dependente do Tempo numa Placa Plana pela Combinação do Método Espectral e da Transformada de Laplace", Tese de Doutorado, PPGEM, UFRGS, Porto Alegre, RS, 2000.

[2] D. Gottlieb e S.A. Orszag, "Numerical Analysis of Spectral Methods: Theory and Applications", SIAM, Philadelphia, 1977.

[3] C.F. Segatto e M.T. Vilhena, The state-of-the-art the LTS $_{N}$ method, Mathematics and Computation, in "Proccedings of the International Conference in Reactor Physics and Environmental Analysis in Nuclear Applications", Madrid, Vol. 2, pp. 1618-1631, 1999. 
[4] J.V.P. de Oliveira e A.V. Cardona, Solução semi-analítica da equação de transporte dependente do tempo, em "Seleta do XXII CNMAC"(J.M. Balthazar, S.M. Gomes e A.Sri Ranga, eds.), Tendências em Matemática Aplicada e Computacional, Vol. 1, pp. 193-200, SBMAC, 2000.

[5] J.V.P. de Oliveira, A.V. Cardona e M.T. Vilhena, Solution of the onedimensional time-dependent discrete ordinates problem in a slab by the spectral an LTS $_{\mathrm{N}}$ methods, Annals of Nuclear Energy, 29 (2001), 13-20.

[6] J.V.P. de Oliveira et al., Solução Semi-analítica da Equação de Transferência Radiativa Não-linear, em " $8^{\text {th }}$ Brazilian Congress of Thermal Engineering and Sciences" (in Portuguese), Porto Alegre, RS, Brazil, 2000.

[7] J.V.P. de Oliveira et al., A Semi-analytical numerical method for timedependent radiative transfer problems in slab geometry With coherent isotropic scattering, Journal of Quantitative Spectroscopy and Radiative Transfer, (2001), to appear.

[8] M. Abramowitz e I.A. Stegun, "Handbook of Mathematical Functions With Formulas, Graphs and Mathematical Table", Dover Publications, New York, 1998 . 